\title{
Identidad ambiental, sentido de lugar y la percepción del contexto en agricultores
}

\section{[Environmental identity, sense of place, and the perception of socio- environmental conflict among farmers]}

\author{
Carlos Adrián Cruz Jurado (i) \& Cesáreo Estrada Rodríguez (i) \\ Universidad Nacional Autónoma de México
}

\begin{abstract}
Resumen
El objetivo de este estudio fue explorar la identidad ambiental, el sentido de lugar y la percepción del contexto socioambiental en un grupo de agricultores habitantes de la zona patrimonial de Xochimilco en la Ciudad de México. En este contexto los usos y costumbres socioculturales y ambientales tradicionales, discrepan de los procesos político-económicos urbanizadores externos. Una manera de entender los conflictos socioambientales es mediante la teoría de la identidad, a partir de la cual se han desarrollado dos constructos: identidad ambiental y sentido de lugar. Se aplicó un cuestionario a un grupo de agricultores y sus familiares, y se efectuó un análisis de contenido por palabra y categoría. Los resultados se discuten en función de las implicaciones teóricas y su importancia para la gestión de los conflictos socioambientales, para favorecer una planificación urbana sustentable y concordante con la identidad ambiental y el sentido de lugar de sus habitantes.
\end{abstract}

Palabras clave: identidad ambiental, sentido de lugar, significados de lugar, conflicto socioambiental, gestión de áreas naturales.

\begin{abstract}
The purpose of this study was to explore environmental identity, sense of place, and the perception of socioenvironmental context in a group of farmers resident in the heritage zone of Xochimilco, Mexico City. In this borough of CDMX, traditional socio-cultural and environmental practices clash with external politicaleconomic processes that favor urban land use and lifestyles. One way to understand the socioenvironmental conflict is through identity theory, which forms the basis of two socio-psychological constructs: environmental identity and sense of place. We applied an open-ended questionnaire to a group of farmers and their relatives, and their responses were subjected to a content analysis. The results are discussed in terms of the theoretical implications of meanings of place, as well as their importance in the management of socioenvironmental conflict and in ensuring sustainable urban planning that is sensitive to the environmental identity and sense of place of inhabitants.
\end{abstract}

Keywords: environmental identity, sense of place, meanings of place, socio-environmental conflict, management of natural areas.

Contacto: La comunicación sobre este artículo debe ser enviada a Carlos Adrián Cruz Jurado, email carlosadrian.c@comunidad.unam.mx

Financiamiento: Esta investigación contó con apoyo del Consejo Nacional de Ciencia y Tecnología mediante la beca nacional No. 368250 y financiamiento de la Autoridad de la Zona Patrimonio Mundial, Natural y Cultural de la Humanidad de Xochimilco, Tláhuac y Milpa Alta mediante el programa de participación vecinal en la operación de acciones institucionales para la preservación del patrimonio cultural, ecosistemas e infraestructura, concepto del programa No. 4419 (ayudas a actores sociales y personas).

Agradecimientos: A la Mtra. Maricela Irepan, al Lic. David Reyes y al Mtro. Aldo de Gasperin por su apoyo e ideas durante el proceso de análisis de la información. 


\section{INTRODUCCIÓN}

La crisis ambiental global es un fenómeno complejo y multicausal con consecuencias que impactan toda la actividad humana. En particular, el sector agropecuario es uno de los mayores generadores de gases de efecto invernadero al producir alrededor del 24\% de este tipo de gases en el planeta (Panel Intergubernamental de Expertos sobre Cambio Climático, 2014). Otros impactos ambientales de la agricultura son la introducción de especies invasoras, alto consumo de agua, y el uso de agroquímicos (National Coalition Against the Misuse of Pesticides, 2006). Las consecuencias de esto incluyen la degradación de los suelos, la contaminación de las aguas, la pérdida de suelo de conservación y hábitats naturales, las especies endémicas amenazadas o en peligro de extinción, entre otros; lo cual ha hecho emerger conflictos socioambientales locales y a gran escala (Corral-Verdugo, 2010).

Ante dicho panorama, el concepto de sustentabilidad propone conciliar las actividades humanas con la necesidad urgente de conservar los ecosistemas, haciendo un uso racionado de los recursos, de manera que se puedan cubrir las necesidades de las generaciones humanas actuales y futuras (World Commission on Environment and Development, 1987). Esta visión del desarrollo se sustenta en un sistema de creencias generales individuales, denominadas nuevo paradigma de la interdependencia humana (NPIH), consistentes con la idea de que la actividad humana debe integrarse coherente y armónicamente a los procesos y ciclos naturales (Corral-Verdugo, Carrus, Bonnes, Möser, \& Sinha, 2008). Así mismo, el NPIH reconoce la necesidad de imponer límites al desarrollo humano para permitir la regeneración de los recursos naturales, y la necesidad de una orientación cognitiva hacia al futuro que permita anticipar los impactos ambientales de cada conducta (Hernández, Suárez, Corral-Verdugo, \& Hess, 2012).

Por otro lado, la literatura sobre psicología de la conservación y la sustentabilidad reconoce que los vínculos emocionales con la naturaleza son una fuerte motivación para llevar a cabo conductas a favor del ambiente (Roczen, Kaiser, \& Bogner, 2010; Sevillano, Aragonés, \& Schultz, 2010). Esta dimensión ha sido aproximada a través de constructos como conectividad con la naturaleza (Mayer \& Frantz, 2004; Schultz, Shriver, Tabanico, \& Khazian, 2004), empatía o toma de perspectiva natural (Schultz, 2000; Sevillano et al., 2007), inclusión de la naturaleza en el self (Schultz, 2002) y apego natural al lugar (Scannel \& Gifford, 2010). De acuerdo con Mayer y Frantz (2004) la conectividad con la naturaleza es un vínculo afectivo entre la persona y la naturaleza, caracterizado por un sentido de pertenencia igualitaria al ecosistema, y de interdependencia entre el individuo y su ambiente, respecto a su bienestar mutuo.

Por ende, la conectividad con la naturaleza implica un sentido de pertenencia, y a la vez de 
internalización del ambiente natural. Schultz (2002) propone que la inclusión de la naturaleza en el self, es un predictor de las actitudes e intenciones a favor del ambiente. Corraliza y Bethelmy (2011), reportaron que la preocupación ambiental es predicha mediante conectividad con la naturaleza, la inclusión de la naturaleza en el self y la frecuencia de visitas a lugares naturales. Gosling y Williams (2010) hallaron que la conectividad con la naturaleza es un predictor de preocupación ambiental, así como de la intención reportada y la posterior realización de conductas de reforestación, en agricultores. Dada la importancia de las creencias ambientales generales y específicas, en conjunción con los factores afectivos y sociales, en las conductas ambientalmente relevantes, así como en la percepción y actuación en conflictos socioambientales (Stern \& Dietz, 2004), su estudio es necesario en poblaciones y escenarios relevantes en América Latina, dada su importancia ambiental y cultural local, así como los procesos de urbanización que ocurren en dichos territorios, que modifican las condiciones ambientales y los modos de vida. Por este motivo, el presente estudio tiene el objetivo de explorar los significados adquiridos, las experiencias vividas en el lugar, y su relación con la percepción de la problemática socioambiental. Para ello se optó por abordar una comunidad de la zona lacustre de Xochimilco, decretada patrimonio natural y cultural de la humanidad por la Organización de las Naciones Unidas para la Educación, la Ciencia y la Cultura (UNESCO), además de formar parte de los pueblos originarios de la Ciudad de México (Herazo \& Moreno, 2014).

Para cumplir este cometido, se recurre a la teoría de la identidad (Tajfel, 1981; Tajfel \& Turner, 1986) como un marco conceptual que permita comprender la interacción entre los individuos y su medio ambiente (Bonaiuto \& Bonnes, 2000). Específicamente los constructos de identidad ambiental y sentido de lugar, consideran el grado de incorporación del medio en el autoconcepto, y de esta manera ayudan a comprender cómo las personas se perciben a sí mismas como miembros de categorías socioambientales más amplias y por qué toman ciertos roles en el marco de conflictos socioambientales (Opotow \& Brook, 2003). A partir de dichas precisiones, se plantea la siguiente pregunta de investigación: ¿Cómo los significados del lugar y la percepción del contexto socioambiental de la zona lacustre de Xochimilco influyen en la conformación de la identidad ambiental y el sentido del lugar de un grupo de agricultores y sus familiares, residentes de un poblado originario de la zona lacustre de Xochimilco?

Identidad ambiental y sentido de lugar

Mediante los procesos de autocategorización y de comparación social, los seres humanos tienden a identificarse con grupos cuyos valores, creencias y dinámicas de interacción son percibidos como similares (Tajfel \& Turner, 1986). Más aún, existe un sesgo intraindividual a evaluar de manera positiva al grupo al que se pertenece, y negativamente a los grupos externos (Turner, 1985). Estos mismos principios operan en los 
conflictos socioambientales, en los cuales grupos con diferentes valores e intereses sobre una problemática ambiental común, tienden a evaluarse de manera negativa, descalificando y estereotipando, lo cual radicaliza sus posturas, y dificulta el diálogo y el encuentro de puntos medios que permitan llegar a soluciones consensuadas (Opotow \& Brook, 2013).

En este sentido, la identidad ambiental remarca el papel de la naturaleza para la autodefinición del individuo, a partir de sus experiencias cercanas a la naturaleza, la educación ambiental que recibe y los grupos con los que interactúa y se identifica (Clayton \& Opotow, 2003). Por lo tanto, la identidad ambiental se describe como un sentido de conexión con el ambiente natural a partir de experiencias biográficas e influye en los modos en que las personas perciben y actúan en relación a su ambiente inmediato (Clayton, 2003). Una identidad ambiental fuerte se relaciona con valoraciones morales intrínsecas de la naturaleza y otras especies, denominados valores biocéntricos (Stern \& Dietz, 1994), así como con una visión altruista y equitativa de la relación entre las personas y su entorno (Schwartz, 1992). Por lo tanto, parte de la identidad ambiental son las nociones implícitas sobre justicia ambiental, mediante la asignación de valor moral a entes naturales (Clayton \& Opotow, 2003). La identidad ambiental influye sobre la conducta al buscar la congruencia con las normas y valores personales o al señalar y buscar la aprobación del medio social (Brick, Sherman, \& Kim, 2017).

Jia, Soucie, Alisat, Curtin, y Pratt (2017) encontraron que aquellos estudiantes con valores biocéntricos se involucraron de manera activa en actividades ambientales en mayor medida, principalmente por tres motivos: valoración de otras especies, vigilancia del ambiente y disgusto por conductas ambientalmente irresponsables de otros. Hinds y Sparks (2008) hallaron que la identidad ambiental y la conectividad con la naturaleza son predictores de la intención de comprometerse a favor de la conservación natural.

Por otro lado, Lacasse (2016) mostró que cuando las personas cuentan con una identidad ambiental en la que se asumen como ambientalistas, es más probable que amplíen la variedad de conductas proambientales en distintos ámbitos de sus vidas, generando estilos de vida sustentables, mientras que aquellos con baja identidad ambiental se conforman moralmente al hacer únicamente conductas proambientales de bajo impacto.

En el contexto agrícola, Michel-Gillou y Möser (2006) encontraron que los agricultores tanto con prácticas proambientales como con prácticas tradicionales interiorizaron elementos naturales en el self y manifestaron amor y respeto por la tierra, sin embargo, se atribuyeron a sí mismos, menor cantidad de responsabilidad sobre los impactos ambientales del sector agrícola, comparado con los sectores industrial y doméstico. Por otro lado, Groth, Curtis, Mendham, y Toman (2016) evaluaron la identidad ocupacional de diversos propietarios de tierras de cultivo, es decir la medida en qué se identifican con el rol de agricultor, encontrando cuatro perfiles distintos: no agricultores, agricultores por pasatiempo, agricultores de 
medio tiempo y agricultores de tiempo completo. Los resultados indicaron que aquellos con menor identidad de agricultor cuentan más valores biocéntricos internalizados y mayor preocupación por el ambiente local, mientras que los valores de los agricultores de tiempo completo se orientaron hacia la posesión de bienes materiales y económicos. Por su parte, Floress y colaboradores (2017) encontraron que aquellos agricultores preocupados por el bien común, conscientes de los impactos negativos de la actividad agrícola, pero también a favor de la agricultura como negocio, se mostraron más dispuestos a restringir su consumo de agua. Este estudio refleja la noción propuesta en el NPIH, en el sentido de que el bien común y el interés individual no resultan contrapuestos, sino interdependientes.

Por otro lado, el sentido de lugar busca abordar de manera integral las relaciones entre las dimensiones ambientales (natural y construida), culturales y simbólicas, sociales y conductuales que componen la experiencia humana en el lugar (Bones \& Bonaiuto, 2002). Las experiencias vividas (positivas y negativas) y las características físicas del lugar determinan los significados del lugar, los cuales, cuando son internalizados en el autoconcepto, conforman el sentido de lugar (Manzo, 2005; Stedman, 2003). Hummon (1992) se refiere al sentido del lugar para aludir a percepciones, emociones $\mathrm{y}$ significados sobre los lugares. En este sentido, Proshansky (1978) propuso el concepto identidad del lugar para referirse a las creencias, preferencias, sentimientos, valores y metas que definen la identidad individual en función del ambiente físico. Otro constructo relacionado es el de dependencia al lugar, definida como la importancia funcional del lugar en relación con las metas individuales (Stokols \& Shumaker, 1981). También se ha empleado apego al lugar en relación con el vínculo afectivo entre las personas y los lugares en los que viven (Scannel \& Gifford, 2010). Raymond, Brown y Weber (2010) encontraron que el sentido de lugar está conformado por una dimensión psicológica conformada por la identidad y la dependencia al lugar, una social conformada por la importancia asignada a los vínculos familiares y de amistad, y la dimensión ambiental originada en la percepción de los elementos naturales del ambiente.

Stokowski (2002) analiza el sentido del lugar desde una perspectiva crítica, al concebir los lugares como sitios dinámicos que contextualizan la interacción social y reproducen las prácticas sociales, ideologías, relaciones de poder y posiciones políticas subyacentes. Por lo tanto, la experiencia en el lugar se encuentra determinada en buena medida por el ambiente físico y los factores estructurales de orden económico-político, tales como los procesos de urbanización. Stedman (2003) mostró que los elementos físicos arquitectónicos, urbanísticos y paisajísticos del lugar originan los significados del lugar, y estos a su vez conforman el sentido de lugar. Jörgensen y Stedman (2001), por su parte, conceptualizan al sentido de lugar siguiendo el modelo de actitud de Fishbein y Ajzen (1975), por lo que se refiere a una evaluación hacia el lugar basada en las creencias, emociones e intenciones de la persona. 
Gustafson (2001) realizó una serie de entrevistas abiertas y realizó un análisis de contenido, con el objetivo de elaborar un modelo de los significados del lugar. El modelo muestra que existe un continuo triangular conformado por tres dimensiones principales: autoconcepto, ambiente social y ambiente físico. Por ello cada significado del lugar puede ubicarse en alguna de las siguientes categorías: (i) self: significados y emociones relacionados con experiencias personales; (ii) self-otros: relaciones interpersonales significativas para el individuo, así como la capacidad de identificar y ser identificado; (iii) otros: cualidades percibidas de los habitantes sin involucrarse con ellos; (iv) otros-ambiente: la manera en la que los rasgos y las conductas de los habitantes influyen y modifican el ambiente; (v) ambiente natural y construido: las condiciones climáticas, además de elementos naturales y arquitectónicos del paisaje; (vi) ambiente-self: el conocimiento sobre el lugar y las oportunidades conductuales; (vii) self-otros-ambiente: la interacción de las tres dimensiones se ve reflejada principalmente en la cultura y las tradiciones, la capacidad de anonimato y las conductas cívicas. Por otro lado, el mismo autor identificó tres procesos simultáneos mediante los cuales se forma el sentido de lugar: (1) distinción: se refiere a que el lugar sea una unidad territorial identificable y delimitada. (2) valuación: implica una evaluación de las características del lugar. (3) continuidad es la dimensión temporal que incluye las experiencias vividas, los usos, costumbres y la cultura del lugar.
Los significados y el sentido de lugar son relevantes en el ámbito de los conflictos socioambientales dado que diversos estudios han encontrado que influyen activamente en la ejecución de conductas protectoras del medio en contextos rurales (Gosling \& Williams, 2010; Vaske \& Kobrin, 2001), en la violación de leyes ambientales (Hernández, Martín, Ruiz, \& Hidalgo, 2010) y en la aceptación social de proyectos de infraestructura para generación de energía eólica (Devine-Wright, \& Howes, 2010). Vaske y Kobrin (2001) encontraron que la identidad del lugar es mediadora entre la dependencia del lugar y las conductas protectoras del ambiente. Lewicka (2005) confirmó que mayor sentido del lugar conlleva al involucramiento cívico proactivo, ya sea mediante la vía socioafectiva o la vía cultural. En la primera, el apego al lugar se produce a partir del capital social, es decir de los lazos vecinales y de amistad dentro de la comunidad; mientras que la segunda es motivada por el estatus cultural y el interés en las raíces genealógicas.

El Programa de las Naciones Unidas para el Medio Ambiente (2012) distingue entre el valor utilitario de los ecosistemas, correspondiente con los servicios ambientales como alimento, agua potable y regulación del clima; y el valor simbólico manifestado en significados simbólicos y valores morales. Respecto a las políticas de gestión de áreas naturales, Williams y Vaske (2003) señalan que las decisiones no deben de tomarse únicamente con base en valoraciones utilitaristas, también se deben considerar las dimensiones simbólicas y afectivas asociadas al paisaje natural, así como con la cultura y las 
relaciones sociales. En este mismo sentido, Pol (2002) menciona que, en los procesos de gestión ambiental sustentable, es esencial considerar los valores y las prácticas sociales compartidas de cada lugar. Kyle, Graefe, Manning y Bacon (2004) estudiaron como diferentes tipos de usuario evaluaron tramos de la ruta de los Apalaches, encontrando que si bien la identidad y la dependencia del lugar se encuentran relacionadas de forma positiva, aquellos con mayor identidad del lugar tuvieron una evaluación más negativa del mismo y tuvieron una postura a favor de la conservación del área natural, mientras que quienes tuvieron mayor dependencia al lugar realizaron evaluaciones más positivas y apoyaron medidas de desarrollo como la construcción de infraestructura dentro de la reserva.

Escenario del estudio

Como se ha argumentado, el estudio de la identidad ambiental y el sentido del lugar resulta relevante para una gestión sustentable de las áreas naturales y los conflictos socioambientales. Por lo tanto, el escenario de los pueblos originarios de la zona lacustre de Xochimilco, resulta idóneo para el estudio de como los significados del lugar y la percepción del contexto socioambiental conforman la identidad ambiental y el sentido de lugar. Esta zona ha sido uno de los principales productores de alimentos en el valle de México desde épocas prehispánicas, gracias al conjunto de técnicas agrícolas ancestrales conocidas como chinampería, consistentes en un conjunto de islotes colocados sobre canales de agua, en los que se aprovecha de manera holística y sustentable los servicios ambientales del ecosistema (tierra, agua, árboles y sol), generando un menor impacto ambiental (MerlínUribe et al., 2013).

Actualmente, la legislación ambiental de Ciudad de México establece que la zona de canales en Xochimilco es un área natural protegida, por lo que únicamente se permite hacer uso de suelo de infraestructura para actividades agrícolas, mientras que, los usos de suelo habitacional o comercial se encuentran prohibidos (Procuraduría Ambiental y de Ordenamiento Territorial del Distrito Federal, 2011). Sin embargo, los efectos de la urbanización descontrolada se han manifestado en una variedad de conflictos socioambientales que impactan directamente en el deterioro de la calidad ambiental de la zona y la calidad de vida de sus habitantes: cambios en el uso de suelo y asentamientos urbanos irregulares, extracción masiva de agua, uso de agroquímicos, destrucción del hábitat natural e introducción de especies ajenas al ecosistema (Ramos, Cajuste, Flores, \& García, 2001). Los estragos de dicha problemática son evidentes en estudios que confirman la degradación de los suelos de cultivo, la contaminación del agua de los canales y la escasez de agua potable, así como la amenaza crítica a especies endémicas y emblemáticas de la zona como el ajolote (ambystoma mexicanum) (Angeles, Perevochtchikova, \& Carrillo, 2009). Asimismo, este escenario se caracteriza por formar parte de los poblados originarios de Ciudad de México, dado que su origen se remonta 
a la época precolombina, por lo que el lugar conserva rasgos de identidad y de cultura indígena (Herazo \& Moreno, 2014). Los pueblos originarios en la Ciudad de México se caracterizan por un fuerte sentido de identidad y convivencia colectiva, que los caracteriza y distingue del resto de comunidades que forman parte de la ciudad (Canabal-Cristiani, 2005). Al mismo tiempo, este tipo de poblaciones cuenta tanto con rasgos rurales como características urbanas, lo cual se ve reflejado en la infraestructura, servicios, oferta educativa, cultural, laboral, de transporte y mercantil, coexistiendo con los usos y costumbres locales manifestados en las fiestas patronales y ferias, en los valores y patrones de una composición familiar extensa y en la actividad agrícola como fuente adicional o única de ingresos y alimentos (Neira-Orjuela, 2005). Sin embargo, los procesos de urbanización guiados por intereses económico-políticos globalizadores buscan expandirse continuamente, modificando las características del ambiente construido y natural, así como las actividades, tradiciones, usos y costumbres de los locales y sus formas de interactuar (Pol, 2002). Por lo que, en este escenario, la percepción del contexto socioambiental debe incluir la presencia de elementos tradicionales contrapuestos a los procesos urbanizadores externos a la comunidad.

\section{MÉTODO}

Se elaboró un cuestionario de ocho preguntas abiertas que reflejaran los significados del lugar, las experiencias positivas y negativas en el lugar, la percepción de conflicto socioambiental, y las acciones y obstáculos para su resolución. El cuestionario fue aplicado durante el mes de mayo de 2015, a un total de 36 participantes voluntarios (16 hombres y 20 mujeres) pertenecientes a un grupo organizado de agricultores y sus familiares, pertenecientes a la comunidad de Santiago Tulyehualco. Por lo tanto, los participantes ya se conocían previamente a la aplicación y fue posible contactarlos gracias a la colaboración con la Autoridad de la Zona Patrimonio de la Humanidad de Xochimilco, Tlahuac y Milpa Alta.
La edad promedio del grupo fue de 45 años ( $S D=16.5$ años) y el $58 \%$ de los participantes se dedicaban a la agricultura como principal actividad económica. El $61 \%$ de ellos afirmó ser nativo de la comunidad, mientras que el promedio de tiempo de residencia en la comunidad del grupo fue de 38 años $(S D=19.6$ años). Los principales cultivos que sembraban fueron amaranto, maíz, avena y diversas hortalizas. La mayoría contaron con un nivel educativo hasta la secundaria, seguido por aquellos con bachillerato, primaria, carrera técnica, licenciatura y sin estudios. El 58\% afirmaron conocer que su comunidad forma parte de la zona patrimonial de la humanidad y únicamente el 31\% afirmó conocer la totalidad de los poblados que forman parte de la misma. 
La aplicación del cuestionario se hizo en una sola sesión, en el domicilio donde habitualmente se reunían para efectuar sus asambleas. La aplicación del cuestionario tuvo una duración aproximada de 45 minutos. Se realizó grupalmente, proporcionando papel y bolígrafo a los participantes para que contestaran las preguntas, únicamente se auxilió personalmente a algunos debido a su avanzada edad o por razones de incapacidad para leer o escribir.

Posteriormente las respuestas de todos los participantes fueron transcritas textualmente en un procesador de textos, para proceder con el análisis de contenido de las mismas. Mediante el análisis de contenido se buscó extraer las principales dimensiones que conforman los dominios y taxonomías cognitivas de los participantes (Tojar-Hurtado, 2006), en relación con cada conjunto (significados del lugar, experiencias en el lugar, conflictos socioambientales, acciones y obstáculos).

El análisis se centró en los significados literales o manifiestos del texto, tomando como unidades de análisis, por un lado, las categorías conceptuales elaboradas a priori para clasificar los temas del texto, siguiendo las recomendaciones de Ruiz-Olabuenaga (1996) y, por otro lado, el análisis por palabras a partir de su frecuencia y agrupación en conglomerados en base a la correlación de Pearson entre ellas. Así, el análisis por categorías resulta de carácter deductivo, mientras que el análisis por palabras es de carácter inductivo. El análisis fue auxiliado por el software NVivo Plus, versión 11 mediante el cual fue posible visualizar la jerarquía de las categorías a priori en el texto para cada conjunto en función del número de referencias codificadas, y analizar la frecuencia de las palabras en cada conjunto y su visualización en dendrogramas. Esto facilitó el proceso de identificación de taxonomías y dominios cognitivos, y a su vez la interpretación de los resultados del análisis. Las preguntas del cuestionario según dimensión se muestran en el anexo 1.

\section{RESULTADOS}

A partir del análisis de contenido fue posible determinar las categorías más importantes y las palabras más utilizadas y su relación para cada conjunto/dimensión de análisis del texto. Para el conjunto de los significados de la zona patrimonial de Xochimilco, las categorías a priori más referidas fueron referentes a las actividades agrícolas realizadas (e.g., "Sí porque de ahí trabajo y obtengo ingresos para mi familia"), y la cultura manifestada en los usos y costumbres tradicionales de los pobladores (e.g., "Por un lado conservar la zona natural y por otro lado la parte cultural con la que cuenta nuestra comunidad"). A su vez, también reconocieron el valor intrínseco del ecosistema (e.g., "la conservación de la fauna, que no crezca la mancha urbana para preservar lo poco de flora y fauna silvestre que queda") y la interdependencia entre las 
generaciones actuales y futuras para el cuidado y disfrute de la zona patrimonial (e.g., "Porque de eso dependemos nosotros como nuestros hijos"). Por otro lado, las palabras más frecuentemente mencionadas en este conjunto fueron: conservar (63 menciones), zona (50 menciones), importante (25 menciones), natural (23 menciones), cultivo, naturaleza y, tierra (17 menciones cada una), alimentos (15 menciones), patrimonio (14 menciones) y ambiente (13 menciones).
En la figura 1 se muestra el dendrograma, donde se observa claramente cuatro conglomerados de palabras. En el primero se agrupan palabras relacionadas al trabajo agrícola y cultivo de tierras, el segundo refleja elementos naturales, el tercero agrupa palabras que reflejan un sentido de interdependencia humana entre generaciones actuales y futuras, y el cuarto aquellas relacionadas a la conservación del medio ambiente y la cultura tradicional de la comunidad.

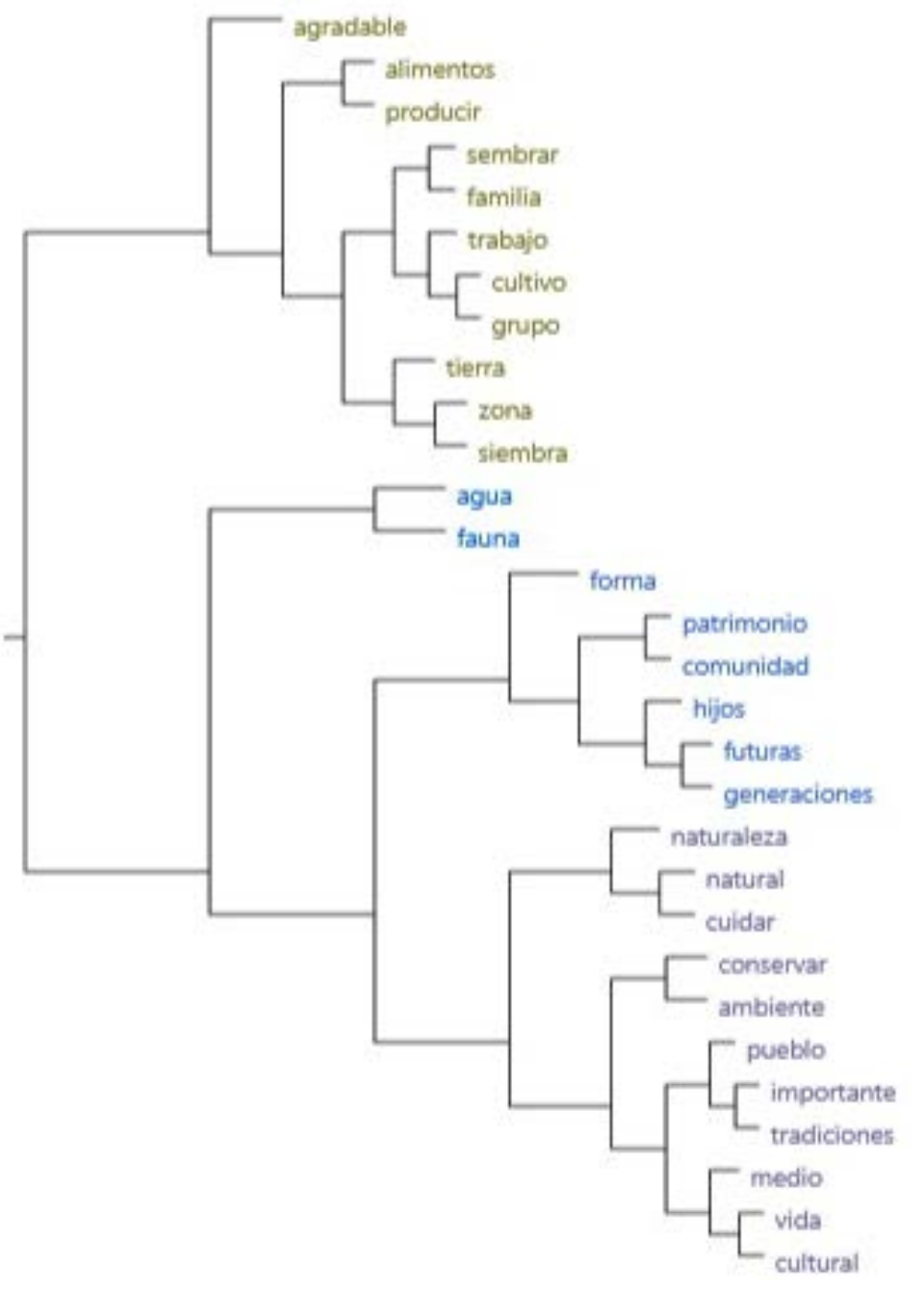

Figura 1. Dendrograma de palabras sobre los significados del lugar. 
A continuación, en el conjunto sobre las experiencias en el lugar, las categorías más referidas fueron positivas: paisaje y medio ambiente (e.g., "Aún tiene zonas naturales como el ejido, con canales, zona cerril y su volcán”), y tradiciones y cultura (e.g., "Es un pueblo con mucha historia, cultura y tradiciones, aún se puede ver gente montada a caballo, buenos deportistas"). Sin embargo, a continuación, las categorías con más referencias fueron de carácter negativo: los otros habitantes (e.g., "El tipo de personas con las que de vez en cuando llego a toparme, son groseras y no les importa dejar su basura en la calle o maltratar a los animales"), tránsito vehicular (e.g., "La contaminación y el tráfico provocado por los coches"), percepción de inseguridad ( e.g., "Que no tengamos seguridad ni para la familia ni para todo Tulyehualco"), contaminación y basura (e.g., "el pueblo ya es muy sucio por tanto habitante que ha llegado"), y espacio público (e.g., "Lo que más me disgusta son las calles mal arregladas y las inundaciones que se hacen y los transportes públicos sobre todo, los moto-taxis"). En el análisis por palabra, aquellas repetidas con más frecuencia fueron: gente (53 menciones), pueblo (46 menciones), falta y zona (25 menciones cada una), tráfico (21 menciones), calles (20 menciones), tradiciones (15 menciones), conservan (14 menciones), autoridades y familia (11 menciones cada una).

En la Figura 2 se muestra la agrupación de las palabras formando cinco conglomerados en los que se diferencian las experiencias positivas y negativas: el primero hace relación a la cultura, el medio ambiente y las actividades agrícolas de la zona patrimonial, mientras que en el segundo se agrupan aquellas que describen el estilo de vida de la comunidad; sin embargo, en el tercero y el quinto se agrupan las palabras relacionadas a los efectos negativos de la urbanización.

En el conjunto sobre el conflicto socio ambiental de la zona patrimonial, las categorías más referidas fueron la contaminación del agua (e.g., "el agua que se llevan a otras partes y nos dejan la contaminada"), los cambios de uso de suelo (e.g., "El crecimiento de la mancha urbana ya que se empiezan a perder estas zonas"), la escasez de agua potable (e.g., "La contaminación y la falta de agua") y la basura en los canales (e.g., "la contaminación de los canales, la baja recarga de los mantos acuíferos"). Las palabras más usadas en este conjunto fueron: contaminación (40 menciones), basura (16 menciones), agua (15 menciones), canales (14 menciones), mancha y urbana (13 menciones cada una), crecimiento (11 menciones), quema e instalación (10 menciones cada una).

En la Figura 3 se muestra el dendrograma de palabras, donde se observa que se agrupan en cinco conglomerados: el primero y el tercero hacen referencia a los impactos ambientales de la actividad agrícola, mientras que el segundo, cuarto y quinto a los efectos negativos e indeseables de la urbanización en la zona patrimonial. 


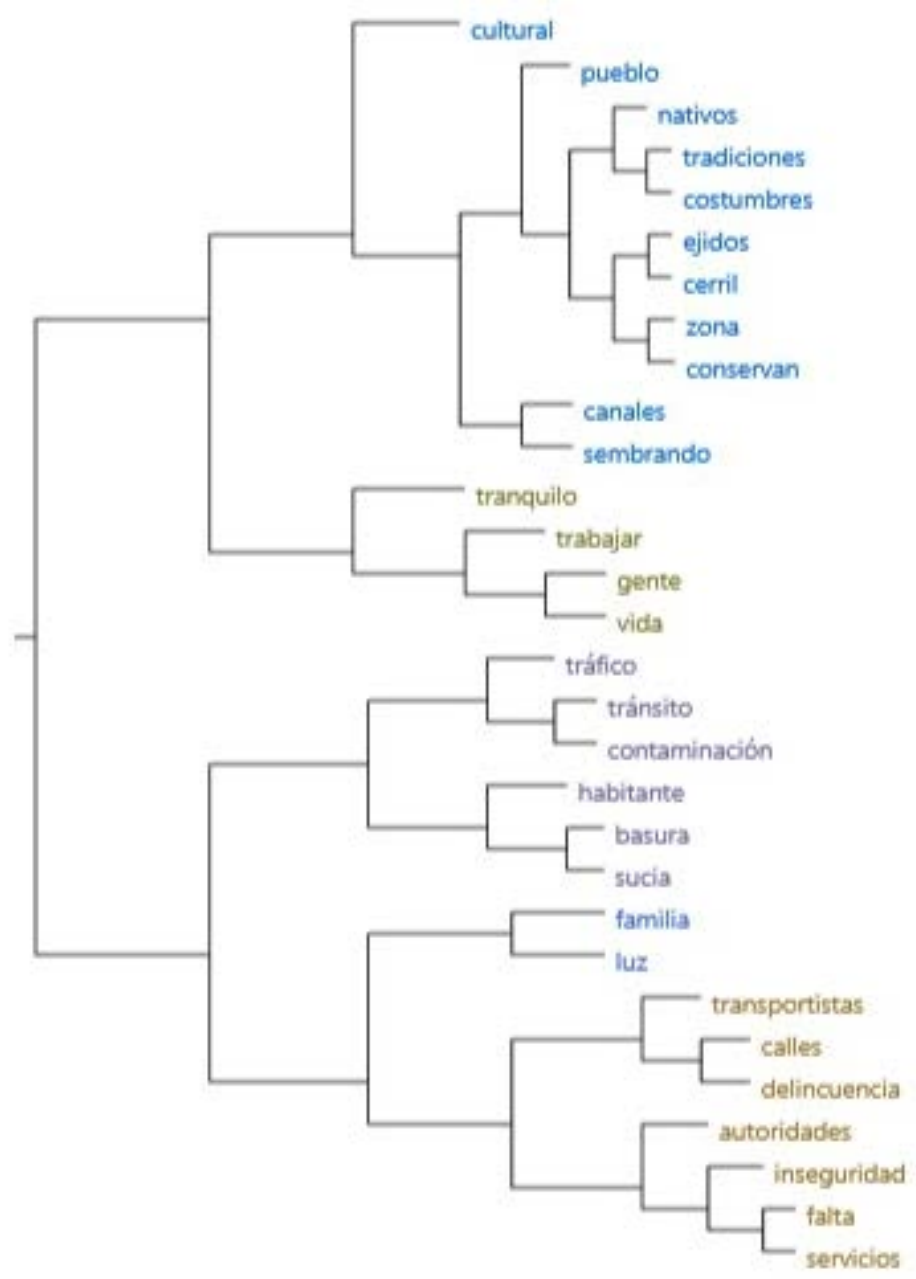

Figura 2. Dendrograma de palabras sobre las experiencias en el lugar.

Por último, en el conjunto sobre las acciones y obstáculos para intervenir ante el conflicto socioambiental, las categorías con mayor referencia fueron en primer lugar las acciones como las prácticas agrícolas ambientalmente responsables (e.g., "Sembrar de manera orgánica y natural, usando implementos que dañen lo menos posible la capa superficial de la tierra que es la que contiene los nutrientes, como el azadón y la yunta") y evitar contaminar y tirar basura (e.g., "Normalmente siempre trato de contribuir en la conservación de los lugares naturales, principalmente no tirando basura en ellos"), reforestación (e.g., "Estamos año con año promoviendo la siembra de árboles, magueyes, captación de agua, ya que es prioritario") y siembra (e.g., "Contribuir al medio ambiente con cosechar y a través de eso ayudar al recargamiento de mantos acuíferos"), seguidas de obstáculos como falta de apoyos gubernamentales (e.g., "No nos dan recursos, se quedan en otras manos y no podemos pagar los gastos, tenemos que hacerlo con nuestras propias manos") y la corrupción (e.g., "Los apoyos 


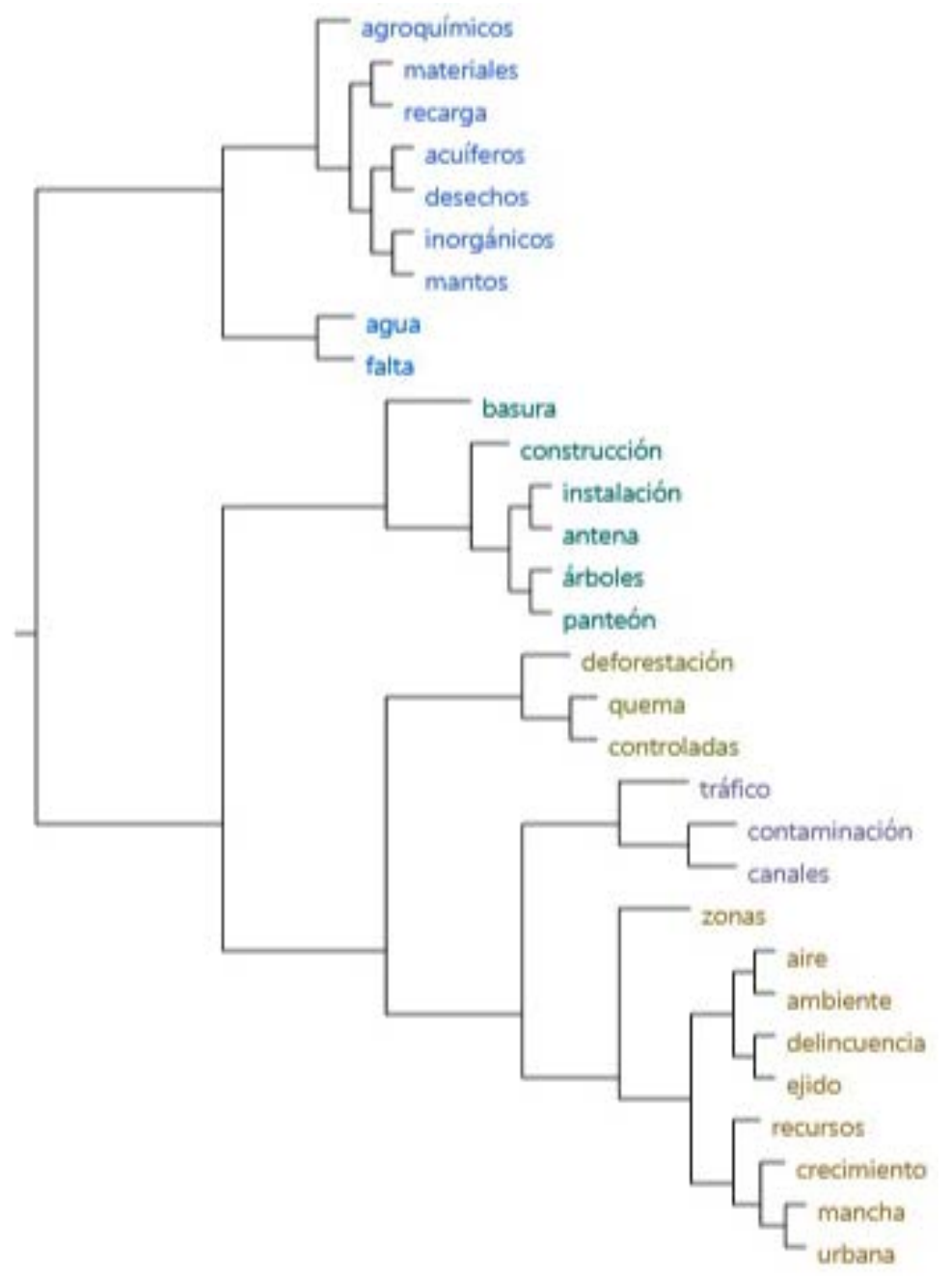

Figura 3. Dendrograma de palabras sobre el conflicto socioambiental

salen etiquetados y para el que realmente trabaja no lo benefician"), además de reconocer también la existencia de problemas al interior del grupo (e.g., "El desacuerdo que en ocasiones el grupo tiene").

Por su parte, el análisis de palabras arrojó que las más usadas fueron: falta y tierra (19 menciones cada una), sembrar (16 menciones), cuidar (15 menciones), abonar (14 menciones), contaminar (13 menciones), recursos (11 menciones), agua y basura (10 menciones cada una).

En la Figura 4, se muestra el dendrograma de palabras, donde se observa como las palabras que hacen alusión a las actividades de conservación de la zona se agrupan en los primeros cuatro conglomerados; mientras que a partir del quinto se agrupan aquellos obstáculos relacionados con la práctica agrícola, y en el sexto las que hacen alusión a los obstáculos relacionados con agentes externos. 


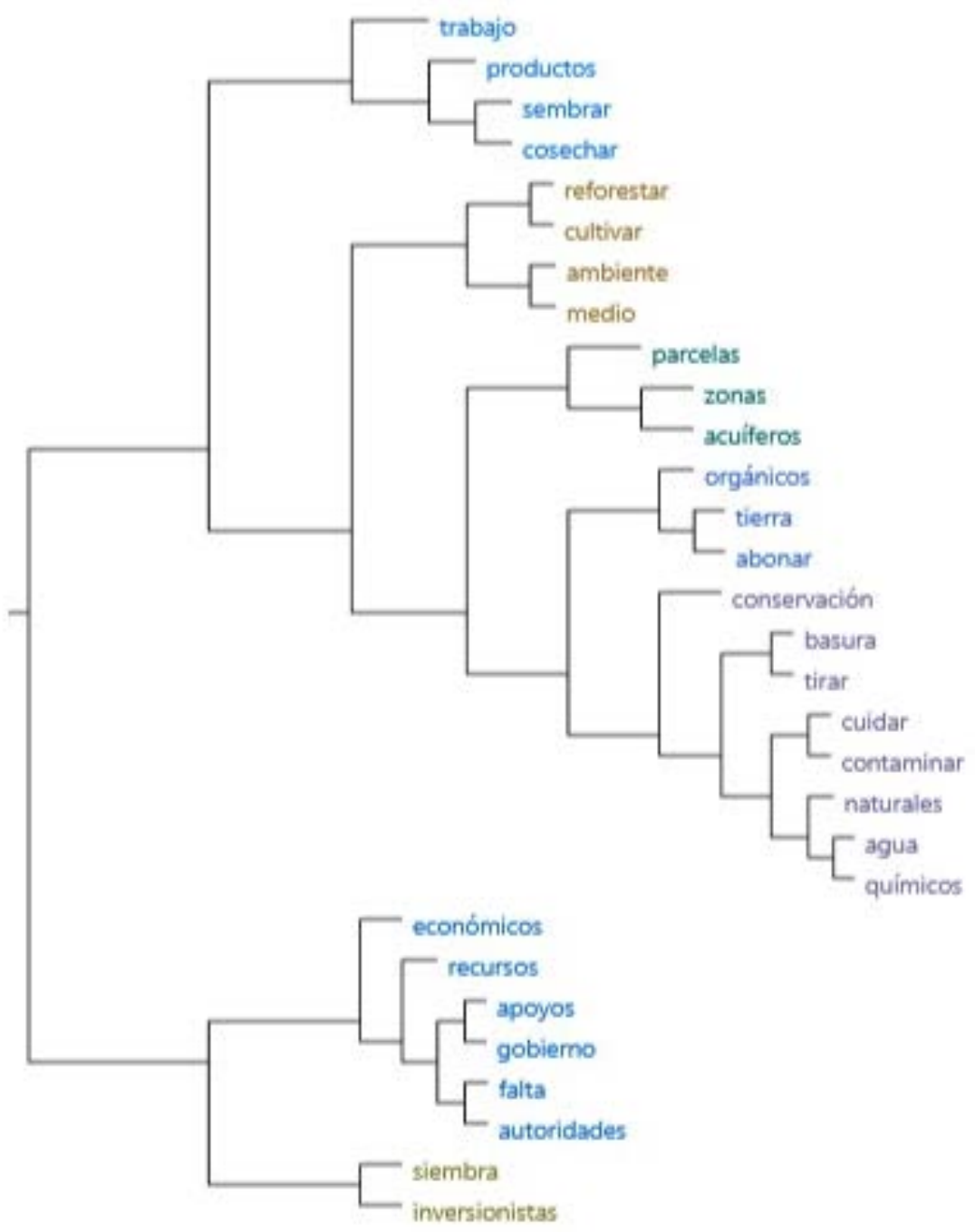

Figura 4. Dendrograma de palabras sobre las acciones y obstáculos ante el conflicto socioambiental

\section{CONCLUSIONES}

Los resultados del análisis de contenido reflejan que los significados del lugar y la percepción del conflicto socioambiental en la zona patrimonial de Xochimilco reflejan de manera dinámica los elementos ambientales, sociales, culturales y político-económicos que forman parte de las experiencias significativas de los participantes. Por un lado, se reconoce la importancia de conservar la zona patrimonial por valoraciones tanto antropocéntricas (agricultura, usos y costumbres tradicionales, servicios ambientales) como biocéntricas (valor intrínseco del 
ecosistema y el paisaje) (Stern \& Dietz, 1994). A su vez, el análisis reveló que los participantes manifiestan creencias consistentes con el NPIH (Corral-Verdugo et al., 2008), al reconocer la importancia que tiene la zona para su propia subsistencia y que la conservación de la zona requiere del esfuerzo conjunto de la población y autoridades para su posterior aprovechamiento y disfrute por las futuras generaciones. Este hallazgo es consistente con los resultados reportados por Floress y colaboradores (2017) en agricultores estadounidenses, quienes encontraron que el interés personal y la importancia percibida del bien común, no son necesariamente intereses contrapuestos, sino interdependientes. De manera que una primera implicación de este trabajo es que la gestión de conflictos socioambientales debe enfatizar la noción de interdependencia entre los actores, dado que la preservación del interés común es la que permitirá mantener las necesidades y valoraciones particulares en el largo plazo.

En segundo lugar, los resultados apuntan a que los elementos socioambientales de la zona patrimonial forman parte central de los significados y experiencias en el lugar, los cuáles sirven para conformar la identidad ambiental y el sentido de lugar de los participantes (Clayton, 2003; Manzo 2005; Stedman, 2003; Stokowski, 2002). A su vez, este resultado es concordante con el modelo de Gustafson (2001), al ubicar a los significados del lugar en un continuo dinámico entre el auto concepto, la interacción social y las cualidades del ambiente generando por un lado, experiencias positivas relacionadas con las actividades agrícolas, los usos y costumbres tradicionales, y el paisaje natural característico de la zona; y por otro experiencias negativas, relacionadas con los efectos negativos de la urbanización como contaminación de los canales, basura, falta de agua, tránsito intenso y hacinamiento, pérdida de tierras de cultivo.

También los significados y las experiencias del lugar reflejaron las características distintivas de la comunidad respecto a otras que forman parte de Ciudad de México y manifestaron un sentido de continuidad temporal reflejado en los usos y costumbres tradicionales. En concordancia con lo anterior, Twigger-Ross y Uzzel (1996) propusieron que la identidad del lugar se construye a partir de un sentido de distinción y autoeficacia. El trabajo agrícola forma parte central de los significados y las experiencias del lugar, así como para la percepción de acciones y obstáculos para resolver el conflicto socioambiental, además de generar un sentido de distinción y autoeficacia, conforman la identidad y la dependencia al lugar. Por lo tanto, la conservación de las tierras de cultivo en la zona patrimonial es necesaria para mantener su identidad y estilo de vida.

En el contexto del conflicto socioambiental de la zona, resulta relevante este hallazgo, dado que, tal como mencionan Devine-Wright y Howes (2010), el mantenimiento de la identidad del lugar es una motivación para rechazar cualquier proyecto que modifique el paisaje y las características ambientales del lugar, así como la interacción social y los estilos de vida característicos, de manera que los procesos urbanizadores presentes en la zona deben ser consecuentes y compatibles con la identidad del 
lugar y las actividades que se realizan en él, de lo contrario se continuará manifestando una resistencia hacia los mismos.

En relación con la percepción del conflicto socioambiental en la zona patrimonial, los resultados muestran de manera clara que los participantes conciben a los procesos de urbanización como una amenaza al paisaje característico, a los estilos de vida, usos y costumbres tradicionales, por ende, a su identidad ambiental y sentido de lugar. Esta percepción también se ve reflejada en las experiencias negativas, las cuales abarcan desde la contaminación del agua de los canales, la escasez de agua potable, la presencia de basura, el mal estado de la infraestructura y el espacio público, los embotellamientos vehiculares que provocan contaminación del aire y auditiva, y la percepción de inseguridad atribuida a personas ajenas a la comunidad o no nativos. En este sentido, Manzo (2005) menciona que las experiencias negativas son un medio que le permite al individuo tomar decisiones que le permitan adaptarse a las exigencias del medio físico y social en el que se encuentra inmerso, sin dejar a un lado aquellas experiencias positivas que originan los significados y el sentido del lugar. Por su parte, Stokowski (2002) advierte que los significados del lugar, tanto positivos como negativos, son un reflejo de los procesos político-económicos subyacentes que impactan y determinan en buena medida la experiencia en el lugar. En este sentido, el estado debe jugar un rol fundamental como ente regulador de los procesos políticos y económicos de urbanización, y para asegurar el cumplimiento de la legislación en materia ambiental, para así asegurar la sostenibilidad de la zona patrimonial.

De nuevo, las prácticas agrícolas forman parte esencial de las acciones percibidas para solucionar el conflicto socio ambiental. Sin embargo, también forma parte de los obstáculos para resolverlo, dado que se reconoce la existencia de prácticas antiecológicas como el uso de agroquímicos, la quema de tierras, la contaminación de los canales y la tira de basura en el campo y las calles. No obstante, los obstáculos más importantes para resolver el conflicto son atribuidos a entes externos como la falta de recursos económicos y apoyos, y la percepción de ineficacia del gobierno para gestionar adecuadamente la zona. Este resultado es consistente con lo reportado por Michel-Gillou y Möser (2006), en el sentido de que los agricultores atribuyeron en mayor medida a factores sociales su nivel de compromiso con el medio ambiente, y en menor medida a sus propias valoraciones y actitudes ambientales. En este sentido, Opotow y Brook (2003) señalan el rol de la identidad ambiental en la gestión del conflicto socioambiental. Dado que las personas tienden a identificarse con grupos de personas en función de su similitud percibida, esto genera un sesgo cognitivo para evaluar positivamente a los miembros del grupo y atribuir características negativas a personas que no forman parte del grupo. En consecuencia, la gestión del conflicto socioambiental en la zona debe de favorecer la atribución intra grupal de responsabilidad. Sin embargo, esto no exime la responsabilidad del estado, como ente regulador externo, para mantener las condiciones necesarias para que 
cada grupo y sector social puedan cooperar entre sí. Cuando la autoridad es percibida como un ente honesto y a favor de la justicia, las personas se muestran dispuestas a cooperar con otros que no conocen (Tyler, 2000) y a reconocerle legitimidad para sancionar a los transgresores (Balliet, Van-Lange, \& Mulder, 2011).

En futuras investigaciones se propone abordar la problemática desde la perspectiva de las diferencias en las preferencias y acciones para resolver el conflicto, en función del tipo de sentido de lugar, como plantean Kyle y colaboradores (2004). Específicamente, sería importante analizar si aquellas personas con mayor identidad del lugar prefieren una gestión orientada hacia la conservación, mientras que aquellos con mayor dependencia del lugar tienen una orientación hacia el desarrollo. Así mismo, se recomienda replicar el estudio de la identidad ambiental y el sentido de lugar en otros grupos de este y otros poblados de la zona patrimonial, dado que el carácter limitado de la muestra de este estudio no permite extrapolar los resultados del mismo a otros grupos o individuos que formen parte de la misma comunidad. Para este cometido, es posible recurrir a métodos mixtos de investigación que permitan estudiar muestras más amplias sin perder la riqueza de la información cualitativa. Otra debilidad del estudio es que al ser instrumento un cuestionario por escrito, no permitió indagar en profundidad las experiencias, percepciones y significados de los participantes. Sin embargo, creemos que el uso simultáneo de una estrategia de análisis deductiva (categorías a priori) y una estrategia inductiva (frecuencia de palabras) permitió incrementar la validez a los resultados de esta investigación.

\section{REFERENCIAS}

Angeles, G., Perevochtchikova, M., \& Carrillo, J. (2008). Posibles controles hidroecológicos de impacto ambiental por la extracción de agua subterránea en Xochimilco, México. Journal of Latin American Geography, 7(1), 39-56. doi: 10.1353/lag.2008.0008

Balliet, D., van Lange, P., \& Mulder, L. (2011). Reward, punishment and cooperation: A meta-analyis. Psychological Bulletin, 1374), 594-615. doi: 10.1037/a0023489

Bonaiuto, M., \& Bonnes, M. (2000). Social psychological approaches in environmentalbehavior studies. Identity theories and the discursive approach. En S. Wapner, J. Demick, T. Yamamoto, \& H. Minami (Eds.), Theoretical perspectives in environmental behavior research: Underlying assumptons, research problems and methodologies (pp. 67-78). New York, NY: Kluwer Academic/Plenum publishers.

Bonnes, M., \& Bonaiuto, M. (2002). Environmental psychology: From spatialphyisical environment to sustainable development. En R. Bechtel \& A. Churchman (Eds.), Handbook of environmental psychology (pp. 28-54). New York, NY: John Wiley and Sons, Inc.

Brick, C., Sherman, D. K., \& Kim H. S. (2017). 'Green to be seen' and 'brown to keep down': Visibility moderates the effect of identity on pro-environmental behavior. Journal of 

Identidad ambiental, sentido de lugar y la percepción del contexto en agricultores, Revista de Sociología, 34(2), 50-71. doi: 10.5354/0719529X.2019.54716

Environmental Psychology, 51(3), 226-238. doi: 10.1016/j.jenvp.2017.04.004

Canabal-Cristiani, B. (2005). Actores ruralurbanos: Proyectos e identidades. En H. Ávila Sánchez (Coor.), Lo urbano rural, ¿nuevas expresiones territoriales? (pp. 161178). Cuernavaca, México: Centro Regional de Investigaciones Multi-disciplinarias, UNAM.

Clayton, S. (2003). Environmental identity: A conceptual and an operational definition. En S. Clayton \& S. Opotow (Eds.), Identity and the natural environment. The psychological significance of nature (pp. 45-66). Cambridge, MA: The MIT Press.

Clayton, S., \& Opotow, S. (2003). Introduction: Identity and the natural environment. En S. Clayton \& S. Opotow (Eds.), Identity and the natural environment. The psychological significance of nature (pp. 1-24). Cambridge, MA: The MIT Press.

Corral-Verdugo, V. (2010). Psicología de la sustentabilidad. Un análisis de lo que nos hace pro ecológicos y pro sociales. Ciudad de México, México: Trillas.

Corral-Verdugo, V., Carrus, G., Bonnes, M., Möser, G., \& Sinha, J. (2008). Environmental beliefs and endorsement of sustainable development principles in water conservation. Toward a new human interdependence paradigm scale. Environment and Behavior, 40(5), 703-725. doi: 10.1177/0013916507308786

Corraliza, J. A., \& Bethelmy, L. C. (2011). Vinculación a la naturaleza y orientación por la sustentabilidad. Revista de Psicología Social, 26(3), 325-336. doi: 10.1174/021347411797361338

Devine-Wright, P., \& Howes, Y. (2010). Disruption to place attachment and the protection of restorative environments: A wind energy case of study. Journal of Environmental Psychology, 30(3), 271-280. doi: 10.1016/j.jenvp.2010.01.008
Fishbein, M., \& Ajzen, I. (1975). Theories of Attitude: An introduction to theory and research. New York, NY: Addison-Wesley.

Floress, K., García de Jalón, S., Church, S., Babin, N., Ulrich-Schad, J., \& Prokopy, L. (2017). Toward a theory of farmer conservation attitudes: Dual interests and willingness to take action to protect water quality. Journal of Environmental Psychology, 53(1), 73-80. doi: 10.1016/j.jenvp.2017.06.009

Gosling, E., \& Williams, K. (2010). Connectedness to nature, place attachment and conservation behavior: Testing conectiveness theory among farmers. Journal of Environmental Psychology, 30(3), 298-304. doi: 10.1016/j.jenvp.2010.01.005

Groth, T., Curtis, A., Mendham, E., \& Toman, E. (2016). Examining the agricultural producer identity: Utilizing the collective occupational identity construct to create a typology of rural landholders in Victoria, Australia. Journal of Environmental Planning and Management, 60(4), 628-646. doi: 10.1080/09640568.2016.1165189

Gustafson, P. (2001). Meanings of place: Everyday experience and theoretical conceptualizations. Journal of Environmental Psychology, 21(1), 5-16. doi: 10.1006/jevp.2000.0185

Herazo, K., \& Moreno, B. (2014). Sentido de comunidad de un pueblo originario: Santa Martha Acatitla (entre los carrizos). Ciudad de México, México: UNAM.

Hernández, B., Martín, A., Ruíz, C., \& Hidalgo, M. (2010). The role of place identity and place attachment in breaking environmental protection laws. Journal of Environmental Psychology, 30(3), 281-288. doi: 10.1016/j.jenvp.2010.01.009

Hernández, B., Suárez, E., Corral-Verdugo, V., \& Hess S. (2012). The relationship beetween social interdependence as an explanation for proenvironmental behavior. Research in Human Ecology, 19(1), 1-10. 

Identidad ambiental, sentido de lugar y la percepción del contexto en agricultores, Revista de Sociología, 34(2), 50-71. doi: 10.5354/0719529X.2019.54716

Hinds, J., \& Sparks, P. (2008). Engaging with the natural environment: The role of affective connection and identity. Journal of Environmental Psychology, 28(2), 109-120. doi: 10.1016/j.jenvp.2007.11.001

Hummon, D. (1992). Community attachment. En I. Altman \& S. Low (Eds.), Place attachement (pp. 253-276). New York, NY: Plenum Press.

Jia, F., Soucie, K., Alisat, S., Curtin, D., \& Pratt, M. (2017). Are environmental issues moral issues? Moral identity in relation to protecting the natural world. Journal of Environmental Psychology, 52(1), 104-111. doi: 10.1016/j.jenvp.2017.06.004

Jörgensen, B., \& Stedman, R. (2001). Sense of place as an attitude: Lakeshore owners attitudes toward their properties. Journal of Environmental Psychology, 21(3), 233-248. doi: 10.1006/jevp.2001.0226

Kyle, G., Graefe, A., Manning, R., \& Bacon J. (2004). Effects of place attachment on user's perceptions of social and environmental conditions in a natural setting. Journal of Environmental Psychology, 24(2), 213-225. doi: $10.1016 /$ j.jenvp.2003.12.006

Lacasse, K. (2016). Don't be satisfied, identify! Streghtening positive spillover by connecting pro-environmental behaviors to an 'environmentalist' label. Journal of Environmental Psychology, 48(1), 149-158. doi: 10.1016/j.jenvp.2016.09.006

Lewicka, M. (2005). Ways to make people active: The role of place attachment, cultural capital, and neighborhood ties. Journal of Environmental Psychology, 25(4), 381-395. doi: 10.1016/j.jenvp.2005.10.004

Manzo, L. (2005). For better or worse: Exploring multiple dimensions of place meaning. Journal of Environmental Psychology, 25(1), 67-86. doi: 10.1016/j.jenvp.2005.01.002

Mayer, F., \& Frantz, C. (2004). The connectedness to nature scale: A measure of individuals feeling in community with nature. Journal of Environmental
Psychology, 24(4), 503-515. doi: 10.1016/j.jenvp.2004.10.001

Merlín-Uribe, Y., Gonzáles-Esquivel, C., Contreras-Hernández, A., Zambrano, L., Moreno-Casasola, P., \& Astier, M. (2013). Environmental and socio-economic sustainability of chinampas (raised-beds) in Xochimilco, Mexico City. International Journal of Agricultural Sustainability, 11(3), 216-233. doi: 10.1080/14735903.2012.726128

Michel-Guillou, E., \& Möser, G. (2006). Commitment of farmers to environmental protection: From social pressure to environmental conscience. Journal of Environmental Psychology, 26(3), 227-235. doi: 10.1016/j.jenvp.2006.07.004

National Coalition Against the Misuse of Pesticides. (2006). Agricultural justice. Recuperado de https://www.beyondpesticides.org/programs /agricultural-justice

Neira-Orjuela, F. (2005). Microempresas agrícolas urbanas y reorganización productiva. Un estudio de caso en hogares de Xochimilco, México. En H. Ávila Sánchez (Ed.), Lo urbano rural, ¿nuevas expresiones territoriales?. Cuernavaca, México: Centro Regional de Investigaciones Multidisciplinarias, UNAM.

$\mathrm{N}$ Vivo for Windows (Versión 11) [Software Informático]. Victoria, Australia: QSR International Pty. Ltd.

Opotow, S., \& Brook, A. (2003). Identity and exclusión in rageland conflict. En S. Clayton $\&$ S. Opotow (Eds.), Identity and the natural environment. The psychological significance of nature (pp. 250-268). Cambridge, MA: The MIT Press.

World Commission on Environment and Development (1987). Report of the World Commission on Environment and Development: Our common future. New York, NY: Oxford University Press. 
Cruz Jurado, C. A, \& Estrada Rodríguez, C. (2019). Identidad ambiental, sentido de lugar y la percepción del contexto en agricultores, Revista de Sociologia, 34(2), 50-71. doi: 10.5354/0719529X.2019.54716

Panel Intergubernamental de Expertos sobre Cambio Climático (2014). Cambio climático 2014. Información de síntesis. Ginebra, Suiza: OMM, PNUMA.

Pol, E. (2002). Environmental management: A perspective from environmental psychology. En R. Bechtel \& A. Churchman (Eds.), Handbook of environmental psychology (pp. 55-84). New York, NY: John Wiley y Sons.

Procuraduría Ambiental y de Ordenamiento Territorial del Distrito Federal. (2011). Distribución espacial de los Asentamientos humanos irregulares ubicados en el suelo de conservación en relación con el proyecto del programa general de ordenamiento ecológico y zonas de valor ambiental del Distrito Federal. Ciudad de México, México: PAOT.

Programa de las Naciones Unidas para el Medio Ambiente. (2012). Economía Verde. Recuperado de: https://www.unenvironment.org/es/exploralos-temas/economia-verde

Proshansky, H. M. (1978). The city and the selfidentity. Environment and Behavior, 10(2), 147-169. doi: 10.1177/0013916578102002

Ramos, R., Cajuste, I., Flores, D., \& García N. (2001). Metales pesados, sales y sodio en suelos de chinampas en México. Agrociencia, 35(4), 385-395.

Raymond, C., Brown, G., \& Weber, D. (2010). The measurement of place attachment: Personal, community and environmental connections. Journal of Environmental Psychology, 30(4), 422-434. doi: 10.1016/j.jenvp.2010.08.002

Roczen, N., Kaiser, F., \& Bogner, F. (2010). Leverage for sustainable change: motivational sources behind ecological behavior. En V. Corral-Verdugo, C. H. García-Cadena, \& M. Frías-Armenta (Eds.), Psychological approaches to sustainability. current trends in theory, research and applications (pp. 109-123). New York, NY: Nova Science Publishers.
Ruiz-Olabuenaga, J. (1996). Metodología de la investigación cualitativa. Bilbao, España: Universidad de Deusto.

Scannel, L., \& Gifford, R. (2010). The relations between natural and civic place attachment and pro-environmental behavior. Journal of Environmental Psychology, 30(3), 289-297. doi: 10.1016/j.jenvp.2010.01.010

Schultz, P. (2000). Empathizing with nature: The effects of perspective taking on concern for environmental issues. Journal of Social ISsues, 56(3), 391-406. doi: 10.1111/00224537.00174

Schultz, P. (2002). Inclusion with nature: The psychology of human-nature relations. En P. Schmuck \& P. W. Schultz (Eds.), Psychology of sustainable development (pp. 61-78). Norwell, MA: Kluwer Academic.

Schultz, P., Shriver, C., Tabanico, J., \& Khazian, A. (2004). Implicit connections with nature. Journal of Environmental Psychology, 24(1), 31-42. doi: 10.1016/S0272-4944(03)00022-7

Sevillano, V., Aragonés, J., \& Schultz, P. (2010). Altruism and beyond: The motivational basis of pro-environmental behavior. En V. Corral-Verdugo, C. H. García-Cadena, \& M. Frías-Armenta (Eds.), Psychological approaches to sustainability. Current trends in theory, research and applications (pp. 161-184). New York, NY: Nova Science Publishers.

Stedman, R. (2003). Is it really just a social construction? The contribution of the physical environment to sense of place. Society and Natural Resources, 16(8), 671685. doi: 10.1080/08941920309189

Stern, P., \& Dietz, T. (1994). The value basis of environmental concern. Journal of Social Issues, 50(3), 65-84. doi: 10.1111/j.15404560.1994.tb02420.x

Stokols, D., \& Shumaker, S. A. (1981). People and places: A transactional view of settings. En J. Harvey (Ed.), Cognition, social behavior and the environment (pp. 441-488). Hillsdale, NJ: Erlbaum. 
Cruz Jurado, C. A, \& Estrada Rodríguez, C. (2019). Identidad ambiental, sentido de lugar y la percepción del contexto en agricultores, Revista de Sociologia, 34(2), 50-71. doi: 10.5354/0719529X.2019.54716

Stokowski, P. (2002). Languages of place and discourses of power: Constructing new senses of place. Journal of Leisure Research. 34(4), 368-382. doi: 10.1080/00222216.2002.11949977

Tajfel, H. (1981). Human groups and social categories. Cambridge, UK: Cambridge University Press.

Tajfel, H., \& Turner, J. (1986). The social identity theory of intergroup behavior. En S. Worchel \& W. Austin (Eds.), The social psychology of intergroup relations (pp. 7-24). Chicago, MI: Nelson-Hall.

Tojar-Hurtado, J. (2006). Investigación cualitativa, comprender y actuar. Madrid, España: Editorial la Muralla.

Turner, J. (1985). Social categorization and the self-concept: A social cognitive theory of group behavior. En E. Lawler (Ed.), Advances in group processes: Theory and research. Greenwich, CT: JAI Press.

Twigger-Ross, C., \& Uzzell, D. (1996). Place and identity processes. Journal of
Environmental Psychology, 16(3), 205-220. doi: 10.1006/jevp.1996.0017

Tyler, T. (2000). Why do people cooperate in groups? Support for structural solutions to social dilemma problems. En M. Van Vugt, M. Snyder, T. Tyler, \& A. Biel (Eds.), Cooperation in modern society. Promoting the welfare of communities, states and organizations (pp. 64-82). New York, NY: Routledge.

Vaske, J., \& Kobrin, K. (2001). Place attachment and environmentally responsible behavior. Journal of Environmental Education, 32(4), 16-21. doi: 10.1080/00958960109598658

Williams D. R., \& Vaske, J. J. (2003). The measurement of place attachment: Validity and generalizability of a psychometric approach. Forest Science, 49(6), 830-848. doi: 10.1093/forestscience/49.6.830

Manuscrito recibido: 01-05-2019 Manuscrito aceptado: 09-10-2019 


\section{ANEXO 1. \\ Tabla de preguntas y dimensiones del análisis.}

\begin{tabular}{ll}
\hline Pregunta & Dimensión \\
\hline $\begin{array}{l}\text { 1. ¿Qué significado personal tiene para usted la zona patrimonial de } \\
\text { Xochimilco? }\end{array}$ & \\
$\begin{array}{l}\text { 2. ¿Para usted, resulta un tema importante la conservación de la } \\
\text { zona patrimonial? ¿Por qué? }\end{array}$ & Significados del lugar \\
$\begin{array}{l}\text { 3. ¿Para su grupo de trabajo, resulta un tema importante la } \\
\text { conservación de la zona patrimonial? ¿Por qué? }\end{array}$ & \\
\hline $\begin{array}{l}\text { 4. ¿Qué es lo que más le gusta a usted de vivir en su comunidad? } \\
\text { 5. ¿Qué es lo que más le disgusta a usted de vivir en su comunidad? }\end{array}$ & Experiencias en el lugar \\
\hline $\begin{array}{l}\text { 6. ¿Cuáles considera usted que son los principales problemas } \\
\text { ambientales de la zona patrimonial? }\end{array}$ & Conflicto socioambiental \\
\hline 7. ¿Qué acciones cotidianas lleva a cabo a favor de la conservación de & \\
la zona patrimonial? & \\
$\begin{array}{l}\text { 8. ¿Cuáles considera usted que son las principales dificultades a las } \\
\text { que se enfrentan los pobladores para realizar acciones por la }\end{array}$ & Acciones y obstáculos \\
conservación de la zona patrimonial? &
\end{tabular}

